\title{
Smartphone Control Robot with Automatic Firing Gun
}

\author{
R.Hema, M.Sundararajan, S.Balaji
}

\begin{abstract}
The Robotics and Automation industries are led in different divisions in everyday life. It is effectively used to satisfy the requirements of varying and enduring requirements. The task is intended to build up a mechanical vehicle utilizing the cell phone for remote activity, joined with camera and firearm. The robot alongside weapon is utilized to shoot consequently in a particular district by identifying the obscure picture of person and different questions through the assistance of camera with night vision capacities. This is somewhat robot is useful to Arm Forces for security framework.
\end{abstract}

Keywords: Smartphone, Robot, Raspberry Pi, Arduino, Bluetooth, Robotic Control, Image Processing.

\section{INTRODUCTION}

The appearance of new fast innovation and the developing PC limit gave reasonable chance to new robot controls and acknowledgment of new strategies for control hypothesis and additionally in security area. This specialized change together with the increased requirement for elite robots, made quicker, more precise with added prudence, employing recent robotic control protocols, drivers and forced rules of computations. This task portrays another arrangement of robot control frameworks and also security framework for Arm Powers, ordinary person and other living life forms.

In this cutting edge condition everyone utilizes advanced cells which are a piece of their everyday life. They utilize all their day by day acts like daily paper perusing, day by day refreshes, interpersonal interaction, and other functions akin to control automation at home, vehicle protection of vehicles, personal body and physical structures, wellbeing support, and so forth has been composed as applications which can be effortlessly introduced in their grasp held PDAs. This task moved toward a mechanical development control trough the PDAs. Our principle objective of this undertaking is:

i. To play out and control a robot through a smart mobile handset, which performs the role of a transmitter that is lined with the robot through Bluetooth.

Revised Manuscript Received on July 22, 2019

R.Hema, Research Scholar, ECE Department, Bharath Institute of Higher Education and Research, Chennai, India. Email: hemrbujradha@gmail.com

M.Sundararajan, Professor, ECE Department, Bharath Institute of Higher Education and Research, Chennai, India. Email: msrajan69@gmail.com

S.Balaji, Assistant Professor, ECE Department, Bharath Institute of Higher Education and Research, Chennai, India. Email: bala.sripathy@gmail.com ii. That robot contains a weapon which will shoot consequently by distinguishing the obscure picture of any individual close to that predefined locale. And furthermore the weapon can ready to pivot by distinguishing the movement of protest through the assistance of PIR sensor.

iii. Image location is finished by the night vision camera through the assistance of picture preparing strategy.

\section{LITERATURE SURVEY}

Development of a Wireless Direction tool based Mobile Robotic steering Scheme.

The framework is proposed by P,Zhan W Siew, C H Wong, B L Chua, K K Teo. The general PC with very much outlined programming Centralized programming directs the handy robot. The smart phone sends summons using Wi-Fi as a communication convention. An IR intelligent workstation helps the robot, affixed to a mobile platform, to maintain a strategic distance from crash. The IR signal indicates the robot to locate the right way without any impediments. The portable controller is a Tablet. Tablets are profoundly practical and adaptable stage to combine with automated independence and route management. Recent research is focused more towards smart phone applications with robots. The present work, demonstrates a handy and mobile robot controlled through a tablet. The remote communication protocol between the tablet and the microcontroller is portrayed.[1],[4]

Protocol based on Bluetooth steering communicative Robot

An Industrial Robotic Arm, proposed by S Delden and A Whigham, can be directed through an Android Bluetooth protocol. It is enable to pick and place and other assorted acts which an ordinary human could not perform. It can also be enabled to direct other robots, associated with, to perform a task. Each robot is organised to execute specific task by self-controlling its own structural arrangements. The user is enabled to communicate with any terminal through this android protocol, to assign a specific task.

Two structures of Robotic arrangements were framed with cyclic execution algorithm. One structure was framed for spot welding line with simple starting phase on to a part of mechanism a model vehicle and then a rapid phase consisting of six more focal points around the model. The second structure was framed to lift, hold the parts from one stage and then place it to the other bed. The structures were developed with "crisis management" scheme for "typical", "ordinary" and "crisis" stop instructions as required for 
any industrial safety. Crisis instruction stops the robot abruptly while the ordinary stop instruction stops the robot allowing it to finish the present task. These Robotic structures were united with Bluetooth-Android protocol to perform the tasks by getting relevant instructions. [3], [5]

\section{A.Direction-finding scheme based on Range for a Mobile} and handy Robot.

Range identifier route framework is proposed by MacMillan, Allen, Marinakis, and Whitesides. The structure was planned to distinguish a hindrance freeway by utilizing the portable robot which detects the obstacle by utilizing ultrasonic transmitter and receiver. The detected obstacles were informed to controlling gadget through the general RFcommunication The scope of the gadget is wider than alternate tasks yet it is old sort of correspondence procedure. The scope of the way is distinguished and are effectively chosen the great way.[3]

\section{B.Android based Bluetooth Scheme for supervising the Temperature of the Robot.}

Raja Lavanya, Vasumathi Priyadharshini, Raj Pandian and T.Maria Jenifer, had proposed an independent robot framework which can determine the earth temperature exchanging the incentive to a php attendant system through the Bluetooth android protocol. [1],[2]

\section{SYSTEM PLAN}

Our proposed framework is partitioned into three fundamental areas i.e. 1. Arduino segment 2. Raspberry pi segment and 3.Cell phone area. Give us a chance to talk about in insight about this.

\section{Arduino section:}

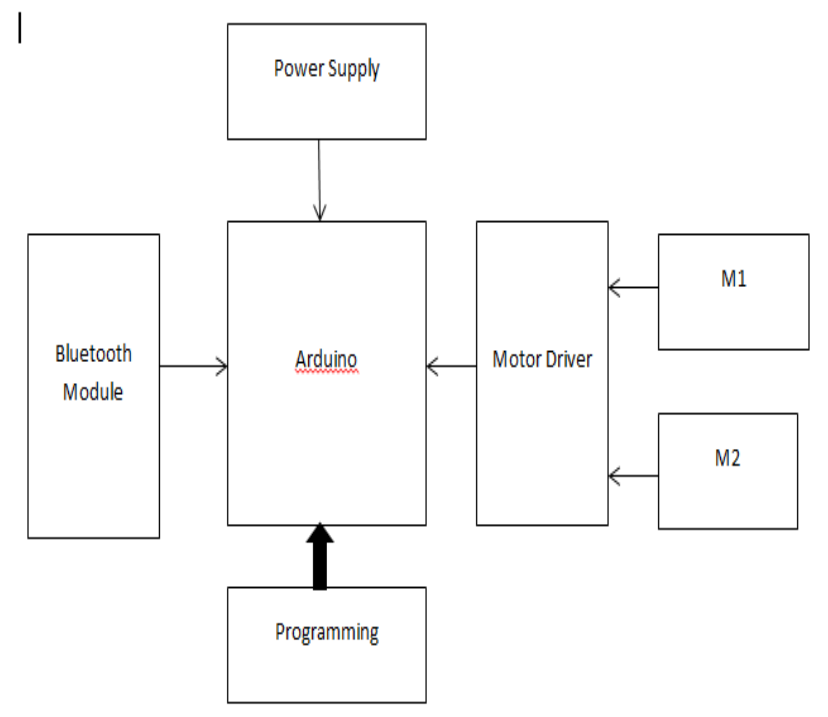

Figure.1: Block diagram of arduino protocol

As appeared in the piece outline, the entire areas are utilized as a recipient as to control the development of the robot through the assistance of Bluetooth application.

\section{Raspberry Pi Module}

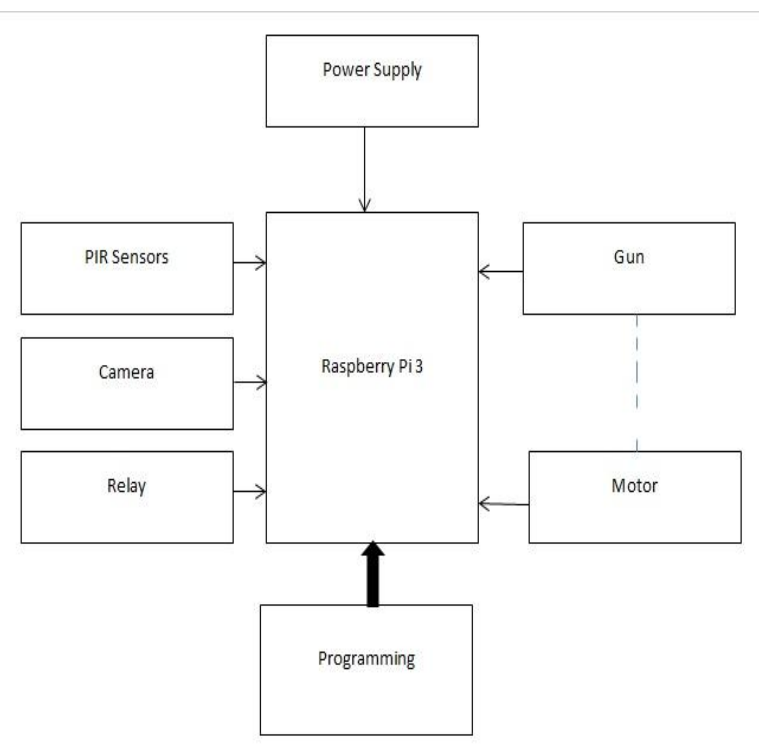

Figure2: Raspberry pi Module

As appeared in the piece graph, the entire area are utilized to play out the programmed shooting task by distinguishing the obscure questions through the assistance of picture handling and furthermore the turn capacity of firearm through the assistance of PIR sensors.

\section{Smartphone section}

Underneath figure is the screen capture of cell phone application which is utilized as a part of this undertaking to control the development of Robot. This area is utilized as a transmitter.

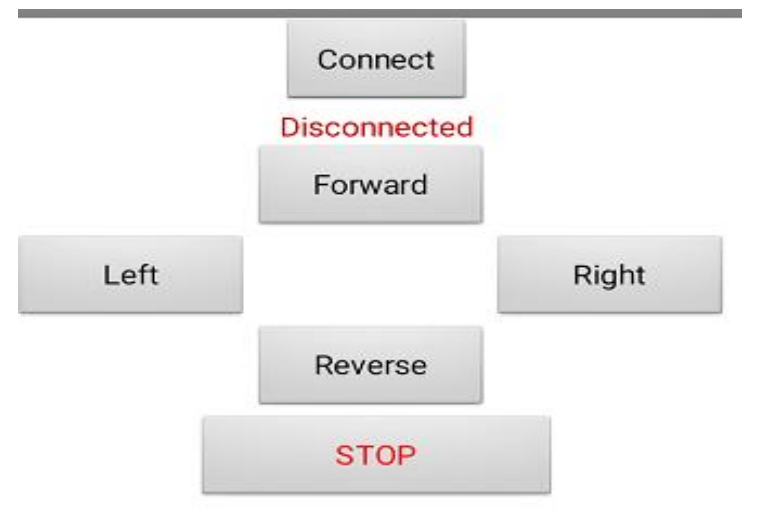

Figure3: Smartphone (Bluetooth) Application.

\section{PROJECT DESCRIPTION}

Here in this robot we have utilized a Bluetooth module which controls the robot by means of 2 100rpm DC Motors. The robot is control by an android phone application. Microcontroller utilized here is Arduino UNO R3 from microcontroller family which contributes in a serial correspondence UART mode and the correspondence is represented on 9800bps to discuss it with the Bluetooth module.

The control through Wireless medium has become common requirement in normal life. By and large huge numbers of the wireless controlled robots utilize RF modules. In any case, our venture for mechanical control makes utilization of Android versatile

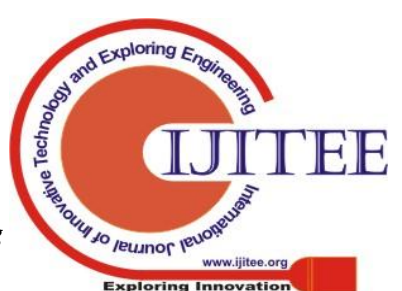


phone which is extremely modest and effectively accessible. The accessible control summonses are more than RFmodules. For this reason the android versatile client needs to introduce a composed application on versatile. At that point the Bluetooth protocol is turned ON. The remote correspondence systems controls the robot. Client can utilize a few summons akin to shift or move forward, backward, left and right through the instructions from the handy Android module. The Robot responds to the Aurdino directions and sends drives the Arduino hardware unit to operate and control the engines. The Arduino UNO at that point exchanges the flag to the engine driver IC's to work the engines.

Other most imperative parts of our undertaking are picture acknowledgment and discovery, revolution of firearm and programmed shooting. On the off chance that the robot discovers objects movement through the assistance of PIR movement location sensor, adjacent their predetermined locale, at that point the weapon and camera setup will be naturally turned focusing on the question. After that the camera will stream picture of the question Raspberry Pi board for picture acknowledgment and discovery reason. As a matter of first importance, Raspberry pi board makes database for the gushing/catching article picture. After that the board will checks the database of already spared picture. In the event that the gushed/caught picture databases are not discovered like the spared database then the Raspberry pi segment will give a flag to the firearm to shoot.If multiple objects detect by the motion sensor and camera, then the Raspberry pi section will give the priority to the nearby object and after that far objects.

Software and Programming Languages: In this venture we are utilizing the accompanying sorts of software and programming dialects.

1. Arduino IDE: to program the Microcontroller of Arduino board by utilizing $\mathrm{C}$ programming dialect. [10]

2. Raspbian: to program Raspberry pi board by utilizing Python programming dialect.[9]

3. Open CV: to make the database of caught picture by utilizing Python programming dialect. [12]

4. Proteous Demonstration: to configuration circuits.[11]

\section{Advantages}

1. Deals with multiple issues, for example, arrangement will spare the life of Arm Forces.

2 . It can control in any confined region from safe places through the assistance of remote control framework i.e. Bluetooth.

\section{Disadvantages}

1. Processing speed might be less if different picture identified by the camera and movement by the PIR sensor.

2. Power Consumption is high.

\section{CONCLUSION AND FUTURE SCOPE}

We are going to complete the first phase of this project by normal image recognition and detection. In future it can be developed to many ideas such as Advanced Image Recognition/Detection, Automatic Robot Control by obstacle avoiding or self-detecting etc. etc.

- Implementation of Solar power in power supply area.

- Our plan is to approach DRDO.

\section{REFERENCES}

1. Surbhi Verma, “Android App Controlled Bluetooth Robot” International Journal of Computer Applications (0975 - 8887) Volume 152 - No.9, October 2016 .

2. Manisha, B.Bansode, Prof.J.K.Singh, “Android Mobile Phone Controlled Wi-Fi Robot" International Journal of Advanced Research in Electronics and Communication Engineering (IJARECE) Volume 4, Issue 6, June 2015

3. SauravBiswas, UmaimaRahman, AsokeNath , "A New Approach to Control a Robot using Android Phone and Colour Detection Technique" SauravBiswas et al, / (IJCSIT) International Journal of Computer Science and Information Technologies, Vol. 6 (3) , 2015, 2985-2989.

4. M.Selvam,"Smart Phone Based Robotic Control For Surveillance Applications" International Journal of Research in Engineering and Technology eISSN: 2319-1163 | pISSN: 2321-7308

5. RowjatulZannatEshita, TanwyBarua, ArzonBarua, AnikMahamood Dip, "Bluetooth Based Android Controlled Robot", American Journal of Engineering Research (AJER) e-ISSN: 2320-0847 p-ISSN : 2320-0936 Volume-5, Issue-3, pp-195-199

\section{AUTHORS PROFILE}

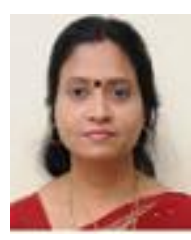

R.Hema, Research Scholar, ECE Department, Bharath Institute of Higher Education and Research, Chennai, India.

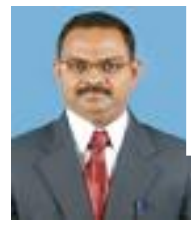

M.Sundararajan, Professor, ECE Department, Bharath Institute of Higher Education and Research, Chennai, India

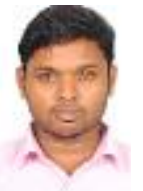

Balaji .S , Assistant Professor, ECE, Bharath Institute of Higher Education and Research,Chennai,India 